\title{
A Family of Integrable Differential-Difference Equations: Tri-Hamiltonian Structure and Lie Algebra of Vector Fields
}

\author{
Ning Zhang' and Xi-Xiang Xu $\mathbb{B}^{2}$ \\ ${ }^{1}$ Public Course Teaching Department, Shandong University of Science and Technology, Taian 271019, China \\ ${ }^{2}$ College of Mathematics and Systems Science, Shandong University of Science and Technology, Qingdao 266590, China
}

Correspondence should be addressed to Xi-Xiang Xu; xixiang_xu@sohu.com

Received 1 April 2021; Accepted 14 August 2021; Published 30 August 2021

Academic Editor: Rigoberto Medina

Copyright (c) 2021 Ning Zhang and Xi-Xiang Xu. This is an open access article distributed under the Creative Commons Attribution License, which permits unrestricted use, distribution, and reproduction in any medium, provided the original work is properly cited.

Starting from a novel discrete spectral problem, a family of integrable differential-difference equations is derived through discrete zero curvature equation. And then, tri-Hamiltonian structure of the whole family is established by the discrete trace identity. It is shown that the obtained family is Liouville-integrable. Next, a nonisospectral integrable family associated with the discrete spectral problem is constructed through nonisospectral discrete zero curvature representation. Finally, Lie algebra of isospectral and nonisospectral vector fields is deduced.

\section{Introduction}

Over the past three decades, the integrable nonlinear differential-difference systems (INDDEs) have received considerable attention. Many INDDEs have been proposed and studied [1-15]. Finding new INDDEs is still an important and difficult work. The discrete zero curvature representation is one of the most significant tools to generate the INDDEs. Furthermore, the Hamiltonian structure of the INDDEs can be established by discrete trace identity or discrete variational identity $[4,5]$. For a family of INDDEs

$$
u_{t_{m}}=K_{n}^{(m)}\left(u_{n}\right), \quad m \geq 0,
$$

one of the interesting problems in the theory of lattice soliton and integrable systems is to look for a Hamiltonian operator $J$ and a sequence-conserved functional $\widetilde{H}_{n}^{(m)},(m \geq 0)$ so that equation (1) may be represented as the following Hamiltonian form:

$$
u_{n, t_{m}}=\frac{\delta \widetilde{H}_{n}^{(m)}}{\delta u_{n}},
$$

where the Hamiltonian functional is $\widetilde{H}_{n}^{(m)}=\sum_{n \in Z} H_{n}^{(m)}(m \geq 0)$. The variational derivative is $\delta \stackrel{H}{n}_{n}^{(m)} / \delta u_{n}=\sum_{n \in Z} E^{-m}\left(\delta H_{n}^{(m)} /\left(\delta u_{n}+m\right)\right),(m \geq 0)$. If we can discover infinitely many involutive conserved functionals for a family of discrete Hamiltonian system, the Liouville integrability of the the discrete Hamiltonian system (2) is proved [9-16].

For a lattice function $f_{n}=f(n)$, the shift operator $E$ and the inverse of $E$ are defined by

$$
\begin{aligned}
E f_{n} & =f_{n+1}, \\
E^{-1} f_{n} & =f_{n-1}, \quad n \in Z .
\end{aligned}
$$

In this paper, we introduce the following spectral problem:

$$
E \varphi_{n}=U_{n}\left(u_{n}, \lambda\right) \varphi_{n}, \quad U_{n}\left(u_{n}, \lambda\right)=\left(\begin{array}{cc}
0 & r_{n} \lambda \\
-s_{n} \lambda & r_{n} s_{n} \lambda^{2}-1
\end{array}\right),
$$

where $\varphi_{n}=\left(\varphi_{n}^{1}, \varphi_{n}^{2}\right)$ is the eigenfunction vector, $\lambda$ is the spectral parameter and $\lambda_{t}=0,\left(r_{n}, s_{n}\right)^{T}$ is the potential vector, 
and $r_{n}=r(n, t), s_{n}=s(n, t)$ depend on integer $n \in Z$ and real $t \in R$. Starting from spectral problem (4), a novel family of INDDEs are deduced. We are going to prove that the obtained family has a triple Hamiltonian structure (tri-Hamiltonian structure). Furthermore, its Louville integrability is presented. It should be pointed out that, in the theory of lattice soliton and integrable systems, the integrable families, which possess concise tri-Hamiltonian structure, are very rare [12-18]. Also, the symmetrical algebraic structure of equations is an important research direction for INDDEs [19-24]. Furthermore, the research of nonisospectral INDDEs has been widespread concern, the algebraic structure of isospectral and nonisospectral vector fields is established in [16-18], and the key of the theory is to derive the corresponding nonisospectral family of INDDEs.

This paper is organized as follows. In Section 2, starting from the matrix spectral problem (4), by means of the discrete zero curvature representation, we derive a family of INDDEs. In Section 3, we establish a triple Hamiltonian structure (triHamiltonian structure) for the obtained integrable family through the discrete trace identity [4]. Infinitely many commuting symmetries and infinitely many commuting conserved functionals for the obtained family are given. The Louville integrability of the obtained family is demonstrated. In Section 4, a nonisospectral integrable family associated with the obtained family is deduced by solving an initial nonisospectral discrete zero curvature equation and the corresponding characteristic operator equation. Lie algebra of isospectral and nonisospectral vector fields is presented. Finally, in Section 5, there will be some conclusions and remarks.

\section{The Family of Integrable Differential- Difference Equations}

In this section, we shall derive a family of integrable differential-difference equations associated with eigenvalue problem (4). To this end, we first solve the following stationary discrete zero curvature equation:

$$
\left(E \chi_{n}\right) U_{n}-U_{n} \chi_{n}=\chi_{n+1} U_{n}-U_{n} \chi_{n}=0 .
$$

Upon setting

$$
\chi_{n}=\left(\begin{array}{cc}
A_{n} & B_{n} \\
C_{n} & -A_{n}
\end{array}\right)
$$

we find that equation (5) becomes

$$
\begin{aligned}
\left(A_{n+1}-A_{n}\right) \lambda^{2} & =\frac{r_{n} C_{n+1}+s_{n} B_{n}}{r_{n} s_{n}} \lambda+\frac{A_{n+1}-A_{n}}{r_{n} s_{n}}, \\
B_{n+1} \lambda^{2} & =-\frac{\left(A_{n+1}+A_{n}\right)}{s_{n}} \lambda+\frac{B_{n+1}}{r_{n} s_{n}}, \\
C_{n} \lambda^{2} & =\frac{A_{n+1}+A_{n}}{r_{n}} \lambda+\frac{C_{n}}{r_{n} s_{n}} .
\end{aligned}
$$

$$
\begin{aligned}
A_{n} & =\sum_{m=0}^{\infty} A_{n}^{(m)} \lambda^{-2 m}, \\
B_{n} & =\sum_{m=0}^{\infty} B_{n}^{(m)} \lambda^{-2 m+1}, \\
C_{n} & =\sum_{m=0}^{\infty} C_{n}^{(m)} \lambda^{-2 m+1},
\end{aligned}
$$

into (7) and comparing each power of $\lambda$ in the equations of (7), we obtain the initial conditions:

$$
\begin{aligned}
\left(A_{n+1}^{(0)}-A_{n}^{(0)}\right) & =0, \\
B_{n+1}^{(0)} & =0, \\
C_{n}^{(0)} & =0,
\end{aligned}
$$

and the recursion relations:

$$
\begin{aligned}
A_{n+1}^{(m+1)}-A_{n}^{(m+1)} & =-\frac{B_{n}^{(m+1)}}{r_{n}}+\frac{C_{n+1}^{(m+1)}}{s_{n}}+\frac{A_{n+1}^{(m)}-A_{n}^{(m)}}{r_{n} s_{n}}, \quad m \geq 0, \\
B_{n+1}^{(m+1)} & =-\frac{A_{n}^{(m)}+A_{n+1}^{(m)}}{s_{n}}+\frac{B_{n+1}^{(m)}}{r_{n} s_{n}}, \quad m \geq 0, \\
C_{n}^{(m+1)} & =\frac{A_{n}^{(m)}+A_{n+1}^{(m)}}{r_{n}}+\frac{C_{n}^{(m)}}{r_{n} s_{n}}, \quad m \geq 0 .
\end{aligned}
$$

Proposition 1. If the initial values are chosen by

$$
\begin{gathered}
A_{n}^{(0)}=\frac{-1}{2}, \\
B_{n}^{(0)}=0,
\end{gathered}
$$

then $A_{n}^{(m)}, B_{n}^{(m)}$, and $C_{n}^{(m)}(m \geq 0)$, which are solved by equation (10), are all local, and they are just rational functions in the two dependent variables $r_{n}$ and $s_{n}$.

Proof. On the basis of second and third equations in equation (10), we see that $B_{n}^{(m+1)}$ and $C_{n}^{(m+1)}$ can be solved locally by $A_{n}^{(m)}, B_{n}^{(m)}$, and $C_{n}^{(m)}(m \geq 0)$. In order to obtain $A_{n}^{(m+1)}(m \geq 0)$ from the first equation in the equation (10), we need to use operator $D^{-1}=(E-1)^{-1}$ to solve the corresponding difference equation. In the following, we are going to show that $A_{n}^{(m+1)}(m \geq 1)$ may be deduced through an algebraic method rather than by solving the difference equations. From (5), we know that

$$
(E-1) \operatorname{tr}\left(\chi_{n}^{2}\right)=2(E-1)\left(A_{n}^{2}+B_{n} C_{n}\right)=0 .
$$

This tells us $\left(A_{n}^{2}+B_{n} C_{n}\right)=\gamma(t)$, where $\gamma(t)$ is an arbitrary function of time variable $t$ only $[10,14]$. Furthermore, we select $\gamma(t)=0$. Then, we obtain a recursion relation for $A_{n}^{(m)}$ :

$$
A_{n}^{(m+1)}=\sum_{j=1}^{m} A_{n}^{(j)} A_{n}^{(m-j+1)}-\sum_{j=1}^{m} B_{n}^{(j)} C_{n}^{(m-j+1)}, \quad m \geq 1 .
$$


Therefore, $A_{n}^{(m+1)}(m \geq 1)$ can be determined locally by $A_{n}^{(m)}, B_{n}^{(m)}, \quad$ and $\quad C_{n}^{(m)}(m \geq 0)$, and then $A_{n}^{(m)}, B_{n}^{(m)}$, and $C_{n}^{(m)}(m \geq 0)$ are all local and they are just rational functions in the two dependent variables $r_{n}$ and $s_{n}$.

The proof is completed.

In particular, we have

$$
A_{n}^{(1)}=-\frac{1}{r_{n} s_{n-1}}, B_{n}^{(1)}=\frac{1}{s_{n-1}}, C_{n}^{(1)}=-\frac{1}{r_{n}}, \ldots
$$

Set

$$
\chi_{n}^{(m)}=\left(\begin{array}{cc}
\sum_{i=0}^{m} A_{n}^{(i)} \lambda^{2 m-2 i} & \sum_{i=0}^{m} B_{n}^{(i)} \lambda^{2 m-2 i+1} \\
\sum_{i=0}^{m} C_{n}^{(i)} \lambda^{2 m-2 i+1} & -\sum_{i=0}^{m} A_{n}^{(i)} \lambda^{2 m-2 i}+A_{n}^{(m)}
\end{array}\right) .
$$

Let us introduce the following auxiliary spectral problems associated with the spectral problem (4):

$$
\varphi_{n_{t_{m}}}=\chi_{n}^{(m)} \varphi_{n}, \quad m \geq 0 .
$$

Then, the compatibility condition of (4) and (16)

$$
\left(E \varphi_{n}\right)_{t_{m}}=E\left(\left(\varphi_{n}\right) t_{m}\right),
$$

is equivalent to the discrete zero curvature equations

$$
U_{n t_{m}}=\left(E \chi_{n}^{(m)}\right) U_{n}-U_{n} \chi_{n}^{(m)}, \quad m \geq 0,
$$

which give rise to the family of integrable differential-difference equations:

$$
\left\{\begin{array}{l}
r_{n_{t_{m}}}=-r_{n} s_{n} B_{n+1}^{(m+1)}-r_{n} A_{n}^{(m)}, \\
s_{n_{t_{m}}}=-r_{n} s_{n} C_{n}^{(m+1)}+s_{n} A_{n+1}^{(m)}, \quad m \geq 0 .
\end{array}\right.
$$

When $m=0$, (19) becomes a trivial linear system:

$$
\left\{\begin{array}{l}
r_{n_{t_{0}}}=-\frac{r_{n}}{2} \\
s_{n_{t_{0}}}=\frac{s_{n}}{2}
\end{array}\right.
$$

And, when $m=2$, we obtain the first INDDE in family (19) as follows:

$$
\left\{\begin{array}{l}
r_{n_{t_{1}}}=-\frac{r_{n}}{r_{n+1} s_{n}}-\frac{1}{s_{n}}, \\
s_{n_{t_{1}}}=\frac{1}{r_{n}}+\frac{s_{n}}{r_{n} s_{n-1}} .
\end{array}\right.
$$

\section{Tri-Hamiltonian Structure}

Next, we shall establish a tri-Hamiltonian structure for integrable family (19). First, we introduce some concepts. The variational derivative, the Gateaux derivative, and the inner product are defined, respectively, by

$$
\frac{\delta H_{n}}{\delta u_{n}}=\sum_{m \in Z} E^{(-m)}\left(\frac{\partial H_{n}}{\partial u_{n+m}}\right),
$$

$$
\begin{aligned}
J^{\prime}\left(u_{n}\right)\left[v_{n}\right] & =\left.\frac{\partial}{\partial \varepsilon} J\left(u_{n}+\varepsilon v_{n}\right)\right|_{\varepsilon=0}, \\
\left\langle f_{n}, g_{n}\right\rangle & =\sum_{n \in Z}\left(f_{n}, g_{n}\right)_{R^{2}},
\end{aligned}
$$

where $f_{n}$ and $g_{n}$ are required to be rapidly vanished at infinity and $\left(f_{n}, g_{n}\right)_{R^{2}}$ denotes the standard inner product of $f_{n}$ and $g_{n}$ in the Euclidean space $R^{2}$. The adjoint operator $J^{*}$ of $J$ is defined by $\left\langle f_{n}, J^{*} g_{n}\right\rangle=\left\langle J f_{n}, g_{n}\right\rangle$. If an operator $J$ has the property $J^{*}=-J$, then $J$ is said to be skew-symmetric. If a skew-symmetric operator $J$ meets the Jacobi identity, i.e.,

$$
\left\langle J^{\prime}\left(u_{n}\right)\left[J f_{n}\right] g_{n}, h_{n}\right\rangle+\operatorname{Cycle}\left(f_{n}, g_{n}, h_{n}\right)=0,
$$

then operator $J$ is called a Hamiltonian operator. Based on a given Hamiltonian operator $J$, we can define a Poisson bracket [4]:

$$
\begin{aligned}
\left\{f_{n}, g_{n}\right\}_{J} & =\left\langle\frac{\delta f_{n}}{\delta u_{n}}, J \frac{\delta g_{n}}{\delta u_{n}}\right\rangle=\sum_{n \in Z}\left\langle\frac{\delta f_{n}}{\delta u_{n}}, J \frac{\delta g_{n}}{\delta u_{n}}\right\rangle_{R^{2}}, \\
(1-E)^{-1} & =\frac{1}{2}\left(\sum_{k=0}^{\infty} E^{k}-\sum_{k=-\infty}^{-1} E^{k}\right), \\
\left(1-E^{-1}\right)^{-1} & =\frac{1}{2}\left(\sum_{k=-\infty}^{-1} E^{k+1}-\sum_{k=0}^{\infty} E^{k+1}\right) .
\end{aligned}
$$

Following [4], we set

$$
S_{n}=\chi_{n}\left(U_{n}\right)^{-1}=\left(\begin{array}{cc}
\frac{s_{n} B_{n} \lambda+A_{n}\left(r_{n} s_{n} \lambda^{2}-1\right)}{r_{n} s_{n} \lambda^{2}} & -\frac{A_{n}}{s_{n} \lambda} \\
\frac{-s_{n} A_{n} \lambda+C_{n}\left(r_{n} s_{n} \lambda^{2}-1\right)}{r_{n} s_{n} \lambda^{2}} & -\frac{C_{n}}{s_{n} \lambda}
\end{array}\right),
$$

$\langle Y, Z\rangle$ is defined as $\operatorname{Tr}(Y Z)$, where $Y$ and $Z$ are the some order square matrices. Hence, 


$$
\begin{aligned}
& \left\langle R_{n}, \frac{\partial U_{n}}{\partial \lambda}\right\rangle=-\frac{C_{n}\left(1+r_{n} s_{n} \lambda^{2}\right)}{s_{n} \lambda^{2}}, \\
& \left\langle R_{n}, \frac{\partial U_{n}}{\partial r_{n}}\right\rangle=-\frac{s_{n} A_{n} \lambda+C_{n}}{r_{n} s_{n} \lambda}, \\
& \left\langle R_{n}, \frac{\partial U_{n}}{\partial s_{n}}\right\rangle=\frac{A_{n}-r_{n} \lambda C_{n}}{s_{n}} .
\end{aligned}
$$

Then, the discrete trace identity becomes

$$
\left\{\begin{array}{c}
\frac{\delta}{\delta r_{n}} \sum_{n \in Z}\left\langle S_{n}, \frac{\partial U_{n}}{\partial \lambda}\right\rangle=\lambda^{-\varepsilon} \frac{\partial}{\partial \lambda} \lambda^{\varepsilon}\left\langle S_{n}, \frac{\partial U_{n}}{\partial r_{n}}\right\rangle, \\
\frac{\delta}{\delta s_{n}} \sum_{n \in Z}\left\langle S_{n}, \frac{\partial U_{n}}{\partial \lambda}\right\rangle=\lambda^{-\varepsilon} \frac{\partial}{\partial \lambda} \lambda^{\varepsilon}\left\langle S_{n}, \frac{\partial U_{n}}{\partial s_{n}}\right\rangle .
\end{array}\right.
$$

Substituting expansions $A_{n}=\sum_{m=0}^{\infty} \quad A_{n}^{(m)} \lambda^{-2 m}$, $B_{n}=\sum_{m=0}^{\infty} B_{n}^{(m)} \lambda^{-2 m+1}$, and $C_{n}=\sum_{m=0}^{\infty} C_{n}^{(m)} \lambda^{-2 m+1}$ into (28) and comparing the coefficients of $\lambda^{-2 m-1}$, we arrive at

$$
\left(\begin{array}{c}
\frac{\delta}{\delta r_{n}} \\
\frac{\delta}{\delta s_{n}}
\end{array}\right) \sum_{n \in Z} H_{n}^{(m)}=(\varepsilon-2 m)\left(\begin{array}{c}
-\frac{s_{n} A_{n}^{(m)}+C_{n}^{(m)}}{r_{n} s_{n}} \\
-\frac{s_{n} A_{n+1}^{(m)}+C_{n}^{(m)}}{s_{n}^{2}}
\end{array}\right) .
$$

When $m=0$ in equation (29), through a direct calculation, we find that $\varepsilon=0$. Thus, equation (29) can be written as

$$
\left(\begin{array}{c}
\frac{\delta}{\delta r_{n}} \\
\frac{\delta}{\delta s_{n}}
\end{array}\right) \tilde{H}_{n}^{(m)}=\left(\begin{array}{c}
-\frac{s_{n} A_{n}^{(m)}+C_{n}^{(m)}}{r_{n} s_{n}} \\
-\frac{s_{n} A_{n+1}^{(m)}+C_{n}^{(m)}}{s_{n}^{2}}
\end{array}\right), \quad m \geq 1,
$$

where

$$
\tilde{H}_{n}^{(m)}=\sum_{j=0}^{m}\left(-\frac{r_{n} s_{n} C_{n}^{(m)}+C_{n}^{(m+1)}}{s_{n}}\right), \quad m \geq 1 .
$$

Moreover, we have

$$
\begin{aligned}
u_{n_{t_{m}}}=\left(\begin{array}{c}
r_{n_{t_{m}}} \\
s_{n_{t_{m}}}
\end{array}\right)=\left(\begin{array}{c}
-r_{n} s_{n} B_{n+1}^{(m+1)}-r_{n}\left(A_{n}^{(m)}+A_{n+1}^{(m)}\right), \\
r_{n} s_{n} C_{n}^{(m+1)}+s_{n}\left(A_{n}^{(m)}+A_{n+1}^{(m)}\right)
\end{array}\right)=\Theta\left(\begin{array}{c}
-\frac{s_{n} A_{n}^{(m+1)}+C_{n}^{(m+1)}}{r_{n} s_{n}} \\
-\frac{s_{n} A_{n+1}^{(m+1)}+C_{n}^{(m+1)}}{s_{n}^{2}}
\end{array}\right) \\
= \\
=\left(\begin{array}{c}
-\frac{s_{n} A_{n}^{(m)}+C_{n}^{(m)}}{r_{n} s_{n}} \\
-\frac{s_{n} A_{n+1}^{(m)}+C_{n}^{(m)}}{s_{n}^{2}}
\end{array}\right)=K\left(\begin{array}{c}
-\frac{s_{n} A_{n}^{(m-1)}+C_{n}^{(m-1)}}{r_{n} s_{n}} \\
-\frac{s_{n} A_{n+1}^{(m-1)}+C_{n}^{(m-1)}}{s_{n}^{2}}
\end{array}\right), \quad m \geq 1,
\end{aligned}
$$

where 


$$
\Theta=\left(\begin{array}{ll}
\Theta_{11} & \Theta_{12} \\
\Theta_{21} & \Theta_{22}
\end{array}\right)
$$

where

$$
\begin{aligned}
& \Theta_{11}=r_{n}(1-E)^{-1} r_{n} E s_{n}\left(1-E^{-1}\right)^{-1} r_{n}-r_{n}(1-E)^{-1} \\
& \cdot s_{n} E^{-1} r_{n}\left(1-E^{-1}\right)^{-1} r_{n}+r_{n}(1-E)^{-1} r_{n}^{2} s_{n}-r_{n}^{2} s_{n}\left(1-E^{-1} r_{n}\right)^{-1}, \\
& \Theta_{12}=r_{n}(1-E)^{-1} r_{n} E s_{n}(1-E)^{-1} s_{n}-r_{n}(1-E)^{-1} \\
& \cdot s_{n} E^{-1} r_{n}(1-E)^{-1} s_{n}-r_{n}(1-E)^{-1} r_{n} s_{n}^{2}-r_{n} s_{n}^{2}(1-E)^{-1} s_{n}, \\
& \Theta_{21}=s_{n}\left(1-E^{-1}\right)^{-1} r_{n} E s_{n}\left(1-E^{-1}\right)^{-1} r_{n}-s_{n}\left(1-E^{-1}\right)^{-1} \\
& \cdot s_{n} E^{-1} r_{n}(1-E)^{-1} r_{n}-s_{n}\left(1-E^{-1}\right)^{-1} r_{n}^{2} s_{n}-r_{n} s_{n}^{2}\left(1-E^{-1}\right)^{-1} r_{n}, \\
& \Theta_{22}=s_{n}\left(1-E^{-1}\right)^{-1} r_{n} E s_{n}(1-E)^{-1} s_{n}-s_{n}\left(1-E^{-1}\right)^{-1} s_{n} E^{-1} r_{n} \\
& \cdot\left(1-E^{-1}\right)^{-1} r_{n}-s_{n}\left(1-E^{-1}\right)^{-1} r_{n}^{2} s_{n}+r_{n}^{2} s_{n}(1-E)^{-1} s_{n} \text {, } \\
& J=\left(\begin{array}{cc}
0 & -r_{n} s_{n} \\
r_{n} s_{n} & 0
\end{array}\right) \\
& K=\left(\begin{array}{ll}
K_{11} & K_{12} \\
K_{21} & K_{22}
\end{array}\right) \text {, }
\end{aligned}
$$

with

$$
\begin{aligned}
K_{11}= & -r_{n}\left(1-E^{-1}\right)^{-1} \frac{1}{s_{n}} E \frac{1}{r_{n}}(1-E)^{-1} r_{n}+r_{n}\left(1-E^{-1}\right)^{-1} \\
& \cdot \frac{1}{r_{n}} E^{-1} \frac{1}{s_{n}}(1-E)^{-1} r_{n}-\frac{1}{s_{n}}(1-E)^{-1} r_{n}+r_{n}\left(1-E^{-1}\right)^{-1} \frac{1}{s_{n}}, \\
K_{12}= & -r_{n}\left(1-E^{-1}\right)^{-1} \frac{1}{s_{n}} E \frac{1}{r_{n}}\left(1-E^{-1}\right)^{-1} s_{n}+r_{n}\left(1-E^{-1}\right)^{-1} \frac{1}{r_{n}} E^{-1} \\
& \cdot \frac{1}{s_{n}}\left(1-E^{-1}\right)^{-1} s_{n}-\frac{1}{s_{n}}\left(1-E^{-1}\right)^{-1} s_{n}-r_{n}\left(1-E^{-1}\right)^{-1} \frac{1}{r_{n}}, \\
K_{21}= & -s_{n}(1-E)^{-1} \frac{1}{s_{n}} E \frac{1}{r_{n}}(1-E)^{-1} r_{n}+s_{n}(1-E)^{-1} \\
& \cdot \frac{1}{r_{n}} E^{-1} \frac{1}{s_{n}}(1-E)^{-1} r_{n}+\frac{1}{r_{n}}(1-E)^{-1} r_{n}+s_{n}(1-E)^{-1} \frac{1}{s_{n}}, \\
K_{22}= & -s_{n}(1-E)^{-1} \frac{1}{s_{n}} E \frac{1}{r_{n}}\left(1-E^{-1}\right)^{-1} s_{n}+s_{n}(1-E)^{-1} \frac{1}{r_{n}} E^{-1} \\
& \cdot \frac{1}{s_{n}}\left(1-E^{-1}\right)^{-1} s_{n}+\frac{1}{r_{n}}\left(1-E^{-1}\right)^{-1} s_{n}-s_{n}(1-E)^{-1} \frac{1}{r_{n}} .
\end{aligned}
$$

For three arbitrary constants $\alpha, \beta$, and $\gamma$, it is easy to verify that the operator $M(\alpha, \beta, \gamma)=\alpha \Theta+\beta J+\gamma K$ is a skew-symmetric operator, i.e., $M(\alpha, \beta, \gamma)=-M(\alpha, \beta, \gamma)^{*}$. Furthermore, by a straightforward and lengthy calculation, we can prove that the operator $M(\alpha, \beta, \gamma)$ fulfills the Jacobian identity (22). So, we can get the following proposition.
Proposition 2. For all values of three arbitrary constants $\alpha, \beta$, and $\gamma, M(\alpha, \beta, \gamma)=\alpha \Theta+\beta J+\gamma K$ is a Hamiltonian operator.

Furthermore, we can obtain that integrable family (19) possesses tri-Hamiltonian structure

$$
\Theta \frac{\delta \widetilde{H}_{n}^{(m+1)}}{\delta u_{n}}=J \frac{\delta \widetilde{H}_{n}^{(m)}}{\delta u_{n}}=K \frac{\delta \widetilde{H}_{n}^{(m)}}{\delta u_{n}}, \quad m \geq 1 .
$$

Moreover, from (7), we find the recursion relation

$$
\frac{\delta \widetilde{H}_{n}^{(m+1)}}{\delta u_{n}}=G \frac{\delta \tilde{H}_{n}^{(m)}}{\delta u_{n}},
$$

where

$$
\begin{aligned}
G= & \left(\begin{array}{cc}
G_{11} & G_{12} \\
G_{21} & G_{22}
\end{array}\right), \\
G_{11}= & -\frac{1}{r_{n}}(1-E)^{-1} \frac{1}{s_{n}} E \frac{1}{r_{n}}(1-E)^{-1} r_{n}+\frac{1}{r_{n}}(1-E)^{-1} \\
& \cdot \frac{1}{r_{n}} E \frac{1}{s_{n}}(1-E)^{-1} r_{n}+\frac{1}{r_{n}}(1-E)^{-1} \frac{1}{s_{n}}+\frac{1}{r_{n}^{1} s_{n}}(1-E)^{-1} r_{n}, \\
G_{12}= & -\frac{1}{r_{n}}(1-E)^{-1} \frac{1}{s_{n}} E \frac{1}{r_{n}}\left(1-E^{-1}\right)^{-1} s_{n}+\frac{1}{r_{n}}(1-E)^{-1} \frac{1}{r_{n}} E^{-1} \\
& \cdot \frac{1}{s_{n}}\left(1-E^{-1}\right)^{-1} s_{n}-\frac{1}{r_{n}}(1-E)^{-1} \frac{1}{r_{n}}+\frac{1}{r_{n}^{2} s_{n}}(1-E)^{-1} r_{n}, \\
G_{21}= & \frac{1}{s_{n}}\left(1-E^{-1}\right)^{-1} \frac{1}{s_{n}} E \frac{1}{r_{n}}(1-E)^{-1} r_{n}-\frac{1}{s_{n}}\left(1-E^{-1}\right)^{-1} \frac{1}{r_{n}} \\
& \cdot E^{-1} \frac{1}{s_{n}}(1-E)^{-1} r_{n}-\frac{1}{s_{n}}\left(1-E^{-1}\right)^{-1} \frac{1}{s_{n}}+\frac{1}{r_{n}^{2} s_{n}}(1-E)^{-1} r_{n}, \\
G_{22}= & \frac{1}{s_{n}}\left(1-E^{-1}\right)^{-1} \frac{1}{s_{n}} E \frac{1}{r_{n}}\left(1-E^{-1}\right)^{-1} s_{n}-\frac{1}{s_{n}}\left(1-E^{-1}\right)^{-1} \frac{1}{r_{n}} \\
& \cdot E^{-1} \frac{1}{s_{n}}\left(1-E^{-1}\right)^{-1} s_{n}+\frac{1}{s_{n}}\left(1-E^{-1}\right)^{-1} \frac{1}{r_{n}}+\frac{1}{r_{n} s_{n}^{2}}\left(1-E^{-1}\right)^{-1} s_{n},
\end{aligned}
$$

where $\Phi=G^{*}$ is a recursion operator. By means of the operator $G$, we have

$$
\begin{aligned}
u_{n_{t_{m}}} & =J \frac{\delta \widetilde{H}_{n}^{(m)}}{\delta u_{n}}=J G \frac{\delta \widetilde{H}_{n}^{(m-1)}}{\delta u_{n}}=J G^{2} \frac{\delta \widetilde{H}_{n}^{(m-2)}}{\delta u_{n}}=\cdots=J G^{m} \frac{\delta \widetilde{H}_{n}^{(0)}}{\delta u_{n}} \\
& =\Phi J \frac{\delta \widetilde{H}_{n}^{(m-1)}}{\delta u_{n}}=\cdots=\Phi^{m}\left(J \frac{\delta \widetilde{H}_{n}^{(0)}}{\delta u_{n}}\right) .
\end{aligned}
$$

Now, we would like to prove the Liouville integrability of the discrete Hamiltonian systems (36). It is crucial to show the existence of infinite involutive conserved functionals. 
Proposition 3. $\left\{\widetilde{H}_{n}^{(m)}\right\}_{m=0}^{\infty}$ are conserved functionals of the that is, whole family (19). And, they are in involution in pairs with respect to the Poisson bracket (24).

$$
G^{*} J=J G
$$

So,

Proof. A direct calculation shows

$$
(J G)^{*}=K^{*}=-K=-J G,
$$

$$
\begin{aligned}
\left\{\widetilde{H}_{n}^{(m)}, \widetilde{H}_{n}^{(l)}\right\}_{J} & =\left\langle\frac{\delta \widetilde{H}_{n}^{(m)}}{\delta u_{n}}, J \frac{\delta \widetilde{H}_{n}^{(l)}}{\delta u_{n}}\right\rangle=\left\langle G^{m-1} \frac{\delta \widetilde{H}_{n}^{(1)}}{\delta u_{n}}, J G^{l-1} \frac{\delta \widetilde{H}_{n}^{(1)}}{\delta u_{n}}\right\rangle \\
& =\left\langle G^{m-1} \frac{\delta \widetilde{H}_{n}^{(1)}}{\delta u_{n}}, G^{*} J G^{l-2} \frac{\delta \widetilde{H}_{n}^{(1)}}{\delta u_{n}}\right\rangle=\left\langle G^{m} \frac{\delta \widetilde{H}_{n}^{(1)}}{\delta u_{n}}, J G^{l-2} \frac{\delta \widetilde{H}_{n}^{(1)}}{\delta u_{n}}\right\rangle \\
& =\left\{\widetilde{H}_{n}^{(m+1)}, \widetilde{H}_{n}^{(l-1)}\right\}_{J}=\cdots=\left\{\widetilde{H}_{n}^{(m+l-1)}, \widetilde{H}_{n}^{(1)}\right\}_{J}
\end{aligned}
$$

Repeating the above argumentation, we can get that

$$
\left\{\widetilde{H}_{n}^{(l)}, \widetilde{H}_{n}^{(m)}\right\}_{J}=\left\{\widetilde{H}_{n}^{(m+l-1)}, \widetilde{H}_{n}^{(1)}\right\}_{J} .
$$

$$
\begin{array}{r}
\left\{\widetilde{H}_{n}^{(m)}, \widetilde{H}_{n}^{(l)}\right\}_{J}=0, \quad m, l \geq 1, \\
\left(\widetilde{H}_{n}^{(m)}\right)_{t_{l}}=\left\langle\frac{\delta \widetilde{H}_{n}^{(m)}}{\delta u_{n}}, u_{t_{l}}\right\rangle=\left\langle\frac{\delta \widetilde{H}_{n}^{(m)}}{\delta u_{n}}, J \frac{\delta \widetilde{H}_{n}^{(l)}}{\delta u_{n}}\right\rangle=\left\{\widetilde{H}_{n}^{(m)}, \widetilde{H}_{n}^{(l)}\right\}_{J}=0, \quad m, l \geq 1 .
\end{array}
$$

The proof is finished.

Based on system (39) and Proposition 3, we can obtain the following theorem.

Theorem 1. Each INDDE in family (19) is Liouville-integrable discrete Hamiltonian system and has tri-Hamitonian structure (36).

\section{Lie Algebraic Structure of Vector Fields}

In Section 2, we have discussed the spectral problem (4) in the isospectral case, namely, $\mathrm{d} \lambda / \mathrm{d} t_{m}=0$. Next, we shall investigate the spectral problem (4) in the nonisospectral case. Now, let us suppose that $\mathrm{d} \lambda / \mathrm{d} t_{m}=\lambda^{2 m+1}$ in (4). First, we shall derive a nonisospectral integrable family using the method proposed in $[23,24]$. The nonisospectral initial zero curvature equation

$$
\left(E V_{n}^{(0)}\right) U_{n}-U_{n} V_{n}=U_{n}^{\prime}\left(\sigma_{n}^{(0)}\right)+\lambda \frac{\partial U_{n}}{\partial \lambda},
$$

is presented. In this equation, the corresponding solutions are easily solved as follows:

$$
\begin{gathered}
\sigma_{n}^{(0)}=\left(\begin{array}{c}
-r_{n} \\
-s_{n}
\end{array}\right), \\
V_{n}^{(0)}=\left(\begin{array}{ll}
1 & 0 \\
0 & 1
\end{array}\right) .
\end{gathered}
$$

Now, we introduce the operator equation:

$$
\left(E \Omega_{n}(X)\right) U_{n}-U_{n} \Omega_{n}(X)=U_{n}^{\prime}(\Phi X)+\lambda^{2} \frac{\partial U_{n}}{\partial \lambda}, \quad X=\left(X_{n}^{(1)}, X_{n}^{(2)}\right)^{T},
$$

In the above equation

$$
\Omega_{n}(X)=\left(\begin{array}{ll}
\Omega_{n}^{(11)} & \Omega_{n}^{(12)} \\
\Omega_{n}^{(21)} & \Omega_{n}^{(22)}
\end{array}\right),
$$

here, $\Omega_{n}^{(i j)}(1 \leq i, j \leq 2)$ is the undetermined function. This (47) is called a characteristic operator equation [23, 24]. Through a straightforward computation, the following results are obtained: 


$$
\begin{aligned}
\Omega_{n}^{(11)}(X)= & -(1-E)^{-1} \frac{X_{1}}{r_{n}^{2} s_{n}}-(1-E)^{-1} \frac{X_{2}}{r_{n} s_{n}^{2}}-(1-E)^{-1} \frac{1}{s_{n}} \\
& \cdot E\left(\frac{1}{r_{n}}\left(1-E^{-1}\right)^{-1} \frac{X_{1}}{r_{n}}+\frac{1}{r_{n}}\left(1-E^{-1}\right)^{-1} \frac{X_{2}}{s_{n}}-\frac{X_{2}}{r_{n} s_{n}}\right)-(1-E)^{-1} \\
& \cdot \frac{1}{r_{n}} E^{-1}\left(\frac{1}{s_{n}}(1-E)^{-1} \frac{X_{1}}{r_{n}}+\frac{1}{s_{n}}(1-E)^{-1} \frac{X_{2}}{s_{n}}+\frac{X_{1}}{r_{n} s_{n}}\right), \\
\Omega_{n}^{(12)}(X)= & -E^{-1}\left(-\frac{1}{s_{n}}(1-E)^{-1} \frac{X_{1}}{r_{n}}-\frac{1}{s_{n}}(1-E)^{-1} \frac{X_{2}}{s_{n}}+\frac{X_{1}}{r_{n} s_{n}}\right) \lambda, \\
\Omega_{n}^{(21)}(X)= & \left(\frac{1}{r_{n}}\left(1-E^{-1}\right)^{-1} \frac{X_{1}}{r_{n}}+\frac{1}{r_{n}}\left(1-E^{-1}\right)^{-1} \frac{X_{2}}{s_{n}}-\frac{X_{2}}{r_{n} s_{n}}\right) \lambda, \\
\Omega_{n}^{(22)}(X)= & -\lambda^{2}\left((1-E)^{-1} \frac{X_{1}}{r_{n}}+(1-E)^{-1} \frac{X_{2}}{s_{n}}\right) .
\end{aligned}
$$

Let us set

$$
\begin{aligned}
\sigma_{n}^{(m)} & =\Phi^{m} \sigma_{n}^{(0)}, \quad m \geq 1, \\
V_{n}^{(m)} & =\lambda^{2 m} V_{n}^{(0)}+\sum_{j=1}^{m} \lambda^{2 m-2 j} \Omega_{n}\left(\sigma_{n}^{(j-1)}\right), \quad m \geq 1 .
\end{aligned}
$$

$$
\begin{aligned}
\left(E V_{n}^{(m)}\right) U_{n}-U_{n} V_{n}^{(m)}= & \lambda^{2 m}\left(\left(E V_{n}^{(0)}\right) U_{n}-U_{n} V_{n}^{(0)}\right) \\
& +\sum_{j=1}^{m} \lambda^{2 m-2 j}\left(\left(E \Omega\left(\sigma_{n}^{(j-1)}\right)\right) U_{n}-U_{n}\left(\Omega\left(\sigma_{n}^{(j-1)}\right)\right)\right) \\
= & \lambda^{2 m}\left(U_{n}^{\prime}\left(\sigma_{n}^{(0)}\right)+\lambda \frac{\partial U_{n}}{\partial \lambda}\right)+\sum_{j=1}^{m} \lambda^{2 m-2 j}\left(U_{n}^{\prime}\left(\Phi\left(\sigma_{n}^{(j-1)}\right)\right)-\lambda^{2} U_{n}^{\prime}\left(\Phi\left(\sigma_{n}^{(j-1)}\right)\right)\right) \\
= & \lambda^{2 m}\left(U_{n}^{\prime}\left(\sigma_{n}^{(0)}\right)+\lambda \frac{\partial U_{n}}{\partial \lambda}\right)+\sum_{j=1}^{m} \lambda^{2 m-2 j}\left(U_{n}^{\prime}\left(\sigma_{n}^{(j)}\right)-\lambda^{2} U_{n}^{\prime}\left(\Phi\left(\sigma_{n}^{(j-1)}\right)\right)\right) \\
= & U_{n}^{\prime}\left[\sigma_{n}^{(m)}\right]+\lambda^{2 m+1} \frac{\partial U_{n}}{\partial \lambda} .
\end{aligned}
$$

Thus, we get a integrable family of nonisospectral $\left(\lambda_{t}=\right.$ $\lambda^{m+1}$ ) discrete evolution equations

$$
u_{n_{t_{m}}}=\sigma_{n}^{(m)}, \quad m \geq 0
$$

and based on (51), the integrable family has the following nonisospectral $\left(\lambda_{t}=\lambda^{2 m+1}\right)$ zero curvature representation
Then, we obtain that 


$$
\begin{aligned}
{[K, S] } & =K^{\prime}[S]-S^{\prime}[K], \quad K^{\prime}[S]=\left.\frac{\mathrm{d}}{\mathrm{d} \epsilon}\right|_{\epsilon=0} K(u+\epsilon S), \\
{[V, W] } & =V^{\prime}[S]-W^{\prime}[K]+[V, W]+g V_{\lambda}-f W_{\lambda},[V, W] \\
& =V W-W V,[[f, g]](\lambda)=f^{\prime}(\lambda) g(\lambda)-f(\lambda) g^{\prime}(\lambda) .
\end{aligned}
$$

We start our calculation as follows:

$$
\begin{aligned}
& \left.\chi_{n}^{(m)}\right|_{\left(r_{n}, s_{n}\right)=(\infty, \infty)}=\lambda^{2 m}\left(\begin{array}{cc}
\frac{-1}{2} & 0 \\
0 & \frac{1}{2}
\end{array}\right) \text {, } \\
& \left.V_{n}^{(m)}\right|_{\left(r_{n}, s_{n}\right)=(\infty, \infty)}=\lambda^{2 m}\left(\begin{array}{ll}
1 & 0 \\
0 & 1
\end{array}\right), \\
& \left.K_{n}^{(m)}\right|_{\left(r_{n}, s_{n}\right)=(\infty, \infty)}=0,\left.\rho_{n}^{(l)}\right|_{\left(r_{n}, s_{n}\right)=(\infty, \infty)}=\Phi^{l}, \\
& \left.\rho_{n}^{(0)}\right|_{\left(r_{n}, s_{n}\right)=(\infty, \infty)}=0, \quad l, m \geq 1, \\
& \left.\chi_{n, \lambda}^{(m)}\right|_{\left(r_{n}, s_{n}\right)=(\infty, \infty)}=2 m \lambda^{2 m-1}\left(\begin{array}{cc}
\frac{-1}{2} & 0 \\
0 & \frac{1}{2}
\end{array}\right) \text {, } \\
& V_{n, \lambda}^{(m)}=2 m \lambda^{2 m-1}\left(\begin{array}{ll}
1 & 0 \\
0 & 1
\end{array}\right) .
\end{aligned}
$$

By the above results, we can get

$$
\begin{aligned}
& {\left.\left[\left[\chi_{n}^{(m)}, \chi_{n}^{(l)}\right]\right]\right|_{\left(r_{n}, s_{n}\right)=(\infty, \infty)} }=0 \\
& {\left.\left[\left[\chi_{n}^{(m)}, V_{n}^{(l)}\right]\right]\right|_{\left(r_{n}, s_{n}\right)=(\infty, \infty)} }=\left.2 m \chi_{n}^{(m+l)}\right|_{\left(r_{n}, s_{n}\right)=(\infty, \infty)} \\
& {\left.\left[\left[V_{n}^{(m)}, V_{n}^{(l)}\right]\right]\right|_{\left(r_{n}, s_{n}\right)=(\infty, \infty)}=\left.2(m-l) V_{n}^{(m+l)}\right|_{\left(r_{n}, s_{n}\right)=(\infty, \infty)} }
\end{aligned}
$$

Moreover, it is easy to find that $U_{n}^{\prime}$ is an injective map. Using the above Lie algebra relations, we can arrive at the Lie algebra of vector fields for the isospectal and nonisospectal families (19) and (52) as follows:

$$
\begin{aligned}
{\left[K_{n}^{(m)}, K_{n}^{(l)}\right] } & =0, \\
{\left[K_{n}^{(m)}, \rho_{n}^{(l)}\right] } & =2 m K_{n}^{(m+l)}, \\
{\left[\rho_{n}^{(m)}, \rho_{n}^{(l)}\right] } & =2(m-l) \rho_{n}^{(m+1)} .
\end{aligned}
$$

From equations (58) and (59), we obtain

$$
\left[K_{n}^{(m)},\left[K_{n}^{(m)}, \rho_{n}^{(l)}\right]\right]=0, \quad m, l \geq 1 .
$$

Hence, $\rho_{n}^{(l)}(l \geq 0)$ are all the master symmetries of $u_{n, t_{m}}=K_{n}^{(m)}(m \geq 0)$. Furthermore, the vector field $\tau_{m l}=t\left[K_{n}^{(m)}, \rho_{n}^{(l)}\right]+\rho_{n}^{(l)}(m, l \geq 0)$ is the symmetries of $u_{t_{l}}=K_{m}^{(m)}$. Here,

$$
K_{n}^{(m)}, \tau_{m l}, \quad m, l \geq 0,
$$

constitute a symmetry algebra of Witt type.

\section{Conclusions and Remarks}

In this paper, we have deduced a family of integrable differential-difference equations through the discrete zero curvature equation. With the help of discrete zero curvature equations, we derive a family of INDDEs. A triple Hamiltonian structure (tri-Hamiltonian structure) for the obtained integrable family is established by the discrete trace identity. Louville integrability of the obtained family is proved. Then, a nonisospectral integrable family associated with the obtained family is derived by solving an initial nonisospectral discrete zero curvature equation and the corresponding characteristic operator equation. Ultimately, Lie algebra of isospectral and nonisospectral vector fields is obtained. In addition, there are other problems worth further investigation for the integrable family (9). For example, the inverse scattering transformation, Hamiltonian structures, binary nonlinearization of Lax pairs, and integrable coupling systems by semidirect sums of Lie algebra.

\section{Data Availability}

The data used to support the findings of this study are available from the corresponding author on reasonable request.

\section{Conflicts of Interest}

The authors declare that there are no conflicts of interest regarding the publication of this paper.

\section{Acknowledgments}

This work was supported by the Youth Science Foundation Project of China (Grant no. 11805114).

\section{References}

[1] M. Ablowitz and P. Clarkson, Solitons, Nonlinear Evolution Equations and Inverse Scattering, Cambridge University Press \& Assessment, Cambridge, UK, 1992.

[2] M. Toda, Theory of Nonlinear Lattice, Springer-Verlag, Berlin, Germany, 2nd edition, 1989.

[3] H. Flaschka, "On the toda lattice. II: inverse-scattering solution," Progress of Theoretical Physics, vol. 51, no. 3, pp. 703-716, 1974. 
[4] G. Z. Tu, "A trace identity and its applications to the theory of discrete integrable systems," Journal of Physics A: Mathematical and General, vol. 23, pp. 3903-3922, 1990.

[5] W.-X. Ma, "A discrete variational identity on semi-direct sums of Lie algebras," Journal of Physics A: Mathematical and Theoretical, vol. 40, no. 50, pp. 15055-15069, 2007.

[6] M. Blaszak and K. Marciniak, "R-matrix approach to lattice integrable systems," Journal of Mathematics and Physics, vol. 35, pp. 4661-4682, 1994.

[7] Y. B. Zeng and Y. S. Li, "New symplectic maps: integrability and lax representation," Chinese Annals of Mathematics, vol. 18B, pp. 457-466, 1997.

[8] A. Pickering and Z. N. Zhu, "New integrable lattice hierarchies," Physics Letters A, vol. 349, pp. 439-445, 2006.

[9] W.-X. Ma and X.-X. Xu, "A modified toda spectral problem and its hierarchy of bi-Hamiltonian lattice equations," Journal of Physics A: Mathematical and General, vol. 37, no. 4, pp. 1323-1336, 2004.

[10] W.-X. Ma, X.-X. Xu, and Y. Zhang, "Semidirect sums of Lie algebras and discrete integrable couplings," Journal of Mathematical Physics, vol. 47, no. 5, pp. 53501-53517, 2006.

[11] W. X. Ma and X. G. Geng, "Backlund transformations of soliton systems from symmetry constraints," CRM Proceedings \& Lecture Notes, vol. 29, p. 313, 1999.

[12] D. Zhang and D. Chen, "Hamiltonian structure of discrete soliton systems," Journal of Physics A: Mathematical and General, vol. 35, no. 33, pp. 7225-7241, 2002.

[13] X. X. Xu and Y. F. Zhang, "A hierarchy of Lax integrable lattice equations, liouville integrability and a new integrable symplectic map," Communications in Theoretical Physics, vol. 41, pp. 321-328, 2004.

[14] X.-X. Xu, "Integrable couplings of relativistic Toda lattice systems in polynomial form and rational form, their hierarchies and bi-Hamiltonian structures," Journal of Physics A: Mathematical and Theoretical, vol. 42, no. 39, pp. 395201395222, 2009.

[15] Y.-P. Sun, D.-Y. Chen, and X.-X. Xu, "A hierarchy of nonlinear differential-difference equations and a new Bargmann type integrable system," Physics Letters A, vol. 359, no. 1, pp. 47-51, 2006.

[16] M. McAnally and W. X. Ma, "Two integrable couplings of a generalized D-Kaup-Newell hierarchy and their Hamiltonian and bi-Hamiltonian structures," Nonlinear Analysis-Theory and Methods, vol. 191, no. 13, Article ID 111629, 2020.

[17] L. Jiang, Y. Jin, W.-X. Ma, S. Shen, and H. Zhu, "Novel soliton hierarchies of Levi type and their bi-Hamiltonian structures," Communications in Nonlinear Science and Numerical Simulation, vol. 23, no. 1-3, pp. 388-396, 2015.

[18] S. Manukure, W.-X. Ma, and E. Appiah, "A tri-Hamiltonian formulation of a new soliton hierarchy associated with so (3, R)," Applied Mathematics Letters, vol. 39, pp. 28-30, 2015.

[19] B. Fuchssteiner and W.-X. Ma, "An approach to master symmetries of lattice equations," in Symmetries and Integrability of Difference Equations, P. A. Clarkson and F. W. Nijhoff, Eds., Cambridge University Press, Cambridge, UK, pp. 247-260, 1999.

[20] J.-B. Zhang, Y. Gongye, and W.-X. Ma, "Conservation laws and $\$ \$ \backslash$ tau $\$ \$ \tau$-Symmetry algebra of the gerdjikov-ivanov soliton hierarchy," Bulletin of the Malaysian Mathematical Sciences Society, vol. 43, no. 1, pp. 111-123, 2020.

[21] S. Zhu, S. Shen, Y. Jin, C. Li, and W.-X. Ma, "New soliton hierarchies associated with the real Lie algebra so $(4, \mathrm{R})$," Mathematical Methods in the Applied Sciences, vol. 40, no. 3, pp. 680-698, 2017.
[22] L. Luo, W.-X. Ma, and E. Fan, "An algebraic structure of zero curvature representations associated with coupled integrable couplings and applications to $\tau$-symmetry algebras," International Journal of Modern Physics B, vol. 25, no. 23n24, pp. 3237-3252, 2011.

[23] W.-X. Ma and B. Fuchssteiner, "Algebraic structure of discrete zero curvature equations and master symmetries of discrete evolution equations," Journal of Mathematical Physics, vol. 40, no. 5, pp. 2400-2418, 1999.

[24] K. M. Tamizhmani and W. X. Ma, "Master symmetries from Lax operators for certain lattice soliton hierarchies," Journal of the Physical Society of Japan, vol. 69, pp. 351-361, 2000. 